\title{
Evaluation of the relationship between slow-waves of intracranial pressure, mean arterial pressure and brain tissue oxygen in TBI: a CENTER-TBI exploratory analysis
}

\author{
Frederick A. Zeiler,2,3,4,5 (- Manuel Cabeleira ${ }^{6} \cdot$ Peter J. Hutchinson $^{7} \cdot$ Nino Stocchetti $^{8,9}$ (D) Marek Czosnyka ${ }^{6,10}$. \\ Peter Smielewski ${ }^{6}$. Ari Ercole ${ }^{1}$ - the CENTER-TBI High-Resolution ICU (HR ICU) Sub-Study Participants and \\ Investigators
}

Received: 25 February 2020 / Accepted: 8 May 2020 / Published online: 16 May 2020

(c) The Author(s) 2020

\begin{abstract}
Brain tissue oxygen $\left(\mathrm{PbtO}_{2}\right)$ monitoring in traumatic brain injury (TBI) has demonstrated strong associations with global outcome. Additionally, $\mathrm{PbtO}_{2}$ signals have been used to derive indices thought to be associated with cerebrovascular reactivity in TBI. However, their true relationship to slow-wave vasogenic fluctuations associated with cerebral autoregulation remains unclear. The goal of this study was to investigate the relationship between slow-wave fluctuations of intracranial pressure (ICP), mean arterial pressure (MAP) and $\mathrm{PbtO}_{2}$ over time. Using the Collaborative European NeuroTrauma Effectiveness Research in Traumatic Brain Injury (CENTER-TBI) high resolution ICU sub-study cohort, we evaluated those patients with recorded high-frequency digital intra-parenchymal ICP and $\mathrm{PbtO}_{2}$ monitoring data of a minimum of $6 \mathrm{~h}$ in duration. Digital physiologic signals were processed for ICP, MAP, and $\mathrm{PbtO}_{2}$ slow-waves using a moving average filter to decimate the highfrequency signal. The first 5 days of recording were analyzed. The relationship between ICP, MAP and $\mathrm{PbtO}_{2}$ slow-waves over time were assessed using autoregressive integrative moving average (ARIMA) and vector autoregressive integrative moving average (VARIMA) modelling, as well as Granger causality testing. A total of 47 patients were included. The ARIMA structure of ICP and MAP were similar in time, where $\mathrm{PbtO}_{2}$ displayed different optimal structure. VARIMA modelling and IRF plots confirmed the strong directional relationship between MAP and ICP, demonstrating an ICP response to MAP impulse. $\mathrm{PbtO}_{2}$ slow-waves, however, failed to demonstrate a definite response to ICP and MAP slow-wave impulses. These results raise questions as to the utility of $\mathrm{PbtO}_{2}$ in the derivation of cerebrovascular reactivity measures in TBI. There is a reproducible relationship between slow-wave fluctuations of ICP and MAP, as demonstrated across various time-series analytic techniques. $\mathrm{PbtO}_{2}$ does not appear to reliably respond in time to slow-wave fluctuations in MAP, as demonstrated on various VARIMA models across all patients. These findings suggest that $\mathrm{PbtO}_{2}$ should not be utilized in the derivation of cerebrovascular reactivity metrics in TBI, as it does not appear to be responsive to changes in MAP in the slow-waves. These findings corroborate previous results regarding $\mathrm{PbtO}_{2}$ based cerebrovascular reactivity indices.
\end{abstract}

Keywords Autoregulation $\cdot$ Brain tissue oxygen $\cdot$ Cerebrovascular reactivity $\cdot$ Traumatic brain injury $\cdot$ TBI

\section{Introduction}

Peter Smielewski and Ari Ercole Joint senior authorship on this work.

Electronic supplementary material The online version of this article (https://doi.org/10.1007/s10877-020-00527-6) contains supplementary material, which is available to authorized users.

Frederick A. Zeiler

umzeiler@myumanitoba.ca

Extended author information available on the last page of the article
Brain tissue oxygen $\left(\mathrm{PbtO}_{2}\right)$ monitoring in adult traumatic brain injury (TBI) is emerging as an important adjunct physiologic parameter for intensive care unit (ICU) directed therapies [1-3]. Invasively placed into the brain parenchyma, typically frontal lobe, such devices measure local extracellular partial pressure of oxygen [4, 5]. This signal provides insight into extracellular oxygen diffusion, and has an emerging literature body in adult TBI supporting its various applications. To date, numerous papers have 
supported the association between low $\mathrm{PbtO}_{2}$ measures and worse global outcome in adult TBI [1-3, 6]. Thresholds for $\mathrm{PbtO}_{2}$ have been suggested, with the current threshold of $20 \mathrm{mmHg}$ being investigated in ongoing randomized control trials [2]. Furthermore, Phase II multi-center studies support feasibility of targeting both intracranial pressure (ICP) and $\mathrm{PbtO}_{2}$ thresholds of $20 \mathrm{mmHg}$, using a protocoled approach, with results from this trial supporting improved outcomes for those patients receiving both ICP and $\mathrm{PbtO}_{2}$ directed therapy, versus ICP directed therapy alone [2].

Another suggested application of $\mathrm{PbtO}_{2}$ monitoring is for cerebrovascular reactivity assessments in TBI [7-9]. Some small, mainly single center retrospective work, have derived the oxygen reactivity index (ORx) through the correlation between slow-waves of $\mathrm{PbtO}_{2}$ and either mean arterial pressure (MAP) or cerebral perfusion pressure (CPP) $[7,8]$. This has been conducted in a similar fashion to the ICP-derived pressure reactivity index (PRx). This ORx metric can be derived based on varying window lengths of data (20, 30 or $60 \mathrm{~min}$ ), longer than PRx (routinely $5 \mathrm{~min}$ long), and has literature to support its association with 6-month outcome $[7,8,10]$.

However, ORx has been demonstrated in various studies of co-variance, to have no relation to more standard metrics of cerebrovascular reactivity [11, 12]. It correlates poorly with PRx [11-13], and has no association with any other multi-modal based metric of cerebrovascular reactivity during multi-variate assessments of co-variance [11]. In particular, ORx has no association with ICP and near infrared spectroscopy (NIRS) based cerebrovascular reactivity metrics [11], which, crucially, are the only such metrics to have some experimental evidence to support their ability to measure aspects of the autoregulatory curve [14-17]. Thus, the role of ORx in cerebrovascular reactivity assessment is questionable, given these previous results and the fact that $\mathrm{PbtO}_{2}$ represents a complex balance between oxygen supply, demand and extracellular diffusion [5, 18], not a surrogate measure of variations in cerebral blood volume (CBV) or cerebral blood flow (CBF) which are required for the derivation of continuous cerebrovascular reactivity metrics [19, 20]. Indeed in-silico simulations show that similar $\mathrm{PbtO}_{2}$ may be found for various different combinations of $\mathrm{CBF}$, metabolic rate and diffusion.

Despite this controversy surrounding ORx, this index is still reported as a metric of cerebrovascular reactivity [7-10]. In order to facilitate understanding regarding the role of $\mathrm{PbtO}_{2}$ monitoring in cerebrovascular reactivity assessments, insight into the time-series relationships between ICP, MAP, CPP and $\mathrm{PbtO}_{2}$ slow-waves is crucial. Observations have been already presented in scientific press, indicating that transients of $\mathrm{PbtO}_{2}$ usually follow changes in CPP [21]. The goal of this study is to provide an exploratory analysis into the multi-variate time-series relationships between ICP, MAP and $\mathrm{PbtO}_{2}$ using time-series methodologies in the Collaborative European NeuroTrauma Effectiveness Research in Traumatic Brain Injury (CENTER-TBI) High-Resolution ICU (HR-ICU) sub-study cohort [22].

\section{Methods}

\subsection{Patient population}

All patients from the multi-center CENTER-TBI high resolution ICU monitoring cohort with parenchymal ICP and $\mathrm{PbtO}_{2}$ monitoring, were included in this analysis. Patients with EVD based ICP data were excluded given the interrupted nature of their recordings. These patients were prospectively recruited between January 2015 and December 2017 from 21 centers in the European Union (EU). All patients were admitted to ICU for their TBI during the course of the study, with high frequency digital signals recorded from their ICU monitors during the course of their ICU stay. All patients suffered predominantly from moderate to severe TBI (moderate $=$ Glasgow Coma Score (GCS) 9 to 12 , and severe $=$ GCS of 8 or less). A minority of patients $(n=9)$ were categorised at the time of admission as suffering from less severe TBI, but experienced subsequent early deterioration leading to ICU admission for care and monitoring. All patients in this cohort had invasive ICP monitoring conducted in accordance with the BTF guidelines [6].

\subsection{Ethics}

Data used in these analyses were collected as part of the CENTER-TBI study which had individual national or local regulatory approval; the UK Ethics approval is provided as an exemplar: (IRAS No: 150943; REC 14/SC/1370). The CENTER-TBI study (EC grant 602150) has been conducted in accordance with all relevant laws of the EU if directly applicable or of direct effect and all relevant laws of the country where the Recruiting sites were located, including but not limited to, the relevant privacy and data protection laws and regulations (the "Privacy Law"), the relevant laws and regulations on the use of human materials, and all relevant guidance relating to clinical studies from time to time in force including, but not limited to, the ICH Harmonised Tripartite Guideline for Good Clinical Practice (CPMP/ ICH/135/95) ("ICH GCP") and the World Medical Association Declaration of Helsinki entitled "Ethical Principles for Medical Research Involving Human Subjects". Informed Consent by the patients and/or the legal representative/next of kin was obtained, accordingly to the local legislations, for all patients recruited in the Core Dataset of CENTER-TBI and documented in the e-CRF. 


\subsection{Data collection}

As part of recruitment to the multi-center high resolution ICU cohort of CENTER-TBI, all patients had demographics and injury data prospectively recorded. Similarly, all patients had high frequency digital signals from ICU monitoring recorded throughout their ICU stay, with the goal of initiating recording within $24 \mathrm{~h}$ of ICU admission. All digital ICU signals were further processed (see Signal Acquisition/Signal Processing). For the purpose of providing a description of the population for this study, basic admission demographics and centrally reported computed tomography (CT) variables for the first available $\mathrm{CT}$ of each patient were extracted [23]. They included: age, admission best GCS motor score and pupillary reactivity (bilaterally reactive, unilateral reactive, bilateral unreactive), Marshall CT Classification [24], Rotterdam CT score [25], presence or absence of traumatic subarachnoid haemorrhage (tSAH), extradural hematoma $(\mathrm{EDH})$, pre-hospital hypotension and pre-hospital hypoxia. CENTER-TBI data version 2.1 was accessed for the purpose of this study, via Opal database software [26].

\subsection{Signal acquisition}

Arterial blood pressure (ABP) was obtained through arterial lines connected to pressure transducers. ICP was acquired from an intra-parenchymal strain gauge probe (Codman ICP MicroSensor; Codman \& Shurtleff Inc., Raynham, MA), parenchymal fibre optic pressure sensor (Camino ICP Monitor, Integra Life Sciences, Plainsboro, NJ, United States; https://www.integralife.com/). $\mathrm{PbtO}_{2}$ monitoring occurred via invasive parenchymal monitoring (Licox probe; Integra, Licox Brain Oxygen Monitoring System, Plainboro, $\mathrm{NJ}$ ), typically placed in the frontal lobe. All signals were recorded using digital data transfer or digitized via an A/D converter (DT9803; Data Translation, Marlboro, MA), where appropriate; sampled at frequency of $100 \mathrm{~Hz}(\mathrm{~Hz})$ or higher, using the ICM + software (Cambridge Enterprise Ltd, Cambridge, UK, https://icmplus.neurosurg.cam.ac.uk) or Moberg CNS Monitor (Moberg Research Inc, Ambler, PA, USA, https://www.moberg.com) or a combination of both. Signal artefacts were removed using both manual and automated methods prior to further processing or analysis.

Of note, the level of arterial line zeroing was not available for all patients in the CENTER-TBI HR ICU sub-study cohort. In general, most participating centre's zeroed the arterial line at the level of the tragus. Regardless, for the purpose of this study and the described statistical analyses performed, the level of arterial line zeroing would not influence any of the results, only the magnitude of raw ABP values. The described analysis focuses on time-series relationships between ICP, ABP and $\mathrm{PbtO}_{2}$, for which a scaling error as a result of difference in zeroing applied to $\mathrm{ABP}$, would not influence the statistical relationships over time for analysis conducted on a patient-by-patient basis.

\subsection{Signal processing}

Post-acquisition processing of the above signals was conducted using ICM+(Cambridge Enterprise Ltd, Cambridge, UK, https://icmplus.neurosurg.cam.ac.uk). Ten second moving averages (updated every $10 \mathrm{~s}$ to avoid data overlap) were calculated for all recorded signals: ICP, ABP (which produced MAP), and $\mathrm{PbtO}_{2}$. This moving average filter was applied to decimate the raw signals to a frequency range association with the slow-wave vasogenic response of cerebrovascular reactivity.

The down-sampled and averaged data were output in $10 \mathrm{~s}$ update frequency (i.e. $10 \mathrm{~s}$ time resolution) for the entire recording period. We then limited the data for analysis to the first 5 days of recording, in order to focus on the acute phase commonly associated with cerebral physiologic derangements. All data curation and processing occurred in $\mathrm{R}(\mathrm{R}$ Core Team (2019). R: A language and environment for statistical computing. R Foundation for Statistical Computing, Vienna, Austria. URL https://www.R-project.org/).

\subsection{Time series analysis and statistics}

All statistical analysis was conducted using R and XLSTAT (Addinsoft, New York, NY; https://www.xlstat.com/en/) add-on package to Microsoft Excel (Microsoft Office 15, Version 16.0.7369.1323). For time series modelling first order differenced data was performed, given the non-stationary nature of the native 10 -s resolution data.

\subsection{ICP, MAP and $\mathrm{PbtO}_{2}$ slow-wave time-series structure}

Using 10-s resolution data, the following analysis was conducted for each patient using the first 5 days of recording. The optimal autoregressive integrative moving average (ARIMA) time-series structure was determined for ICP, MAP and $\mathrm{PbtO}_{2}$ for each individual patient using the following methodology, similar to other publications from our group [27-30]. First, autocorrelation function (ACF) and partial autocorrelation function (PACF) plots were produced, and both Augmented Dickey-Fuller (ADF) and Kwiatkowski-Phillips-Schmidt-Shin (KPSS) testing were conducted, confirming non-stationarity of ICP, MAP and $\mathrm{PbtO}_{2}$. First order differencing was then undertaken to remove all trend components, confirming stationarity by repeating the above-mentioned plots and testing. Next, ARIMA models were built for ICP, MAP and $\mathrm{PbtO}_{2}$, keeping the differencing order of 1 (i.e. $\mathrm{d}=1$ ) and varying both the autoregressive and moving average orders (i.e. $\mathrm{p}$ and $\mathrm{q}$, respectively) 
from 0 to 4, through all respective permutations. The AIC and LL were then tabulated for each of these models, for every patient. Using the AIC and LL, the optimal ARIMA structures for ICP, MAP and $\mathrm{PbtO}_{2}$ were compared in each patient, with the lowest AIC and highest LL values indicating superior models. More details surrounding ARIMA modelling of time-series data can be found in the reference literature [31-33]. The general Box-Jenkin's autoregressive moving average (ARMA) structure for ICP can be expressed as follows:

$\mathrm{ICP}_{\mathrm{t}}=\mathrm{c}+\varepsilon_{\mathrm{t}}+\sum_{i=1}^{p} \varphi_{\mathrm{t}-\mathrm{i}} \mathrm{ICP}_{\mathrm{t}-\mathrm{i}}+\sum_{j=1}^{q} \theta_{\mathrm{t}-\mathrm{j}} \varepsilon_{\mathrm{t}-\mathrm{j}}$

where $\mathrm{c}=$ constant, $\mathrm{t}=$ time " $\mathrm{t}$ ", $\mathrm{i}=$ integer, $\mathrm{j}=$ integer, $\mathrm{p}=$ autoregressive order, ICP $=$ intra-cranial pressure, $\mathrm{q}=$ moving average order, $\varphi=$ autoregressive coefficient at time " $\mathrm{t}-\mathrm{i}$ ", $\theta=$ moving average coefficient at time " $\mathrm{t}-\mathrm{j}$ ", $\varepsilon=$ error term.

\subsection{Analysis of slow-wave relationships}

First order differenced ICP, MAP and $\mathrm{PbtO}_{2}$ slow-waves were analyzed in the 10-s resolution data sheets, per patient. The co-variance of slow-waves of ICP versus MAP, $\mathrm{PbtO}_{2}$ versus MAP, and $\mathrm{PbtO}_{2}$ versus ICP, were evaluated using multi-variate vector ARIMA (VARIMA) models. Such models explore the behavior of two time series recorded simultaneously over time and are derived via extending the standard Box-Jenkin's ARIMA models to multi-variate systems. Further description on this technique can be found in the references [31, 32]. The vector autoregressive moving average model (VARMA) of first order difference ICP and MAP can be represented by the following formula:

$\mathrm{Y}_{\mathrm{t}}=\mathrm{C}+\mathrm{E}_{\mathrm{t}}+\sum_{i=1}^{p} A_{\mathrm{t}-\mathrm{i}} \mathrm{Y}_{\mathrm{t}-\mathrm{i}}+\sum_{j=1}^{q} B_{\mathrm{t}-\mathrm{j}} \mathrm{E}_{\mathrm{t}-\mathrm{j}}$

where $\mathrm{C}=$ constant vector, $\mathrm{t}=$ time " $\mathrm{t}$ ", $\mathrm{i}=$ integer, $\mathrm{j}=$ integer, $p=$ VARMA autoregressive order, $Y_{t}=I C P$ or MAP at time $\mathrm{t}, \mathrm{q}=$ VARMA moving average order, $\mathrm{A}=$ autoregressive coefficient matrix at " $\mathrm{t}-\mathrm{i}$ ", $\mathrm{B}=$ moving average coefficient matrix at time " $t-j$ ", $E=$ error term vector.

We employed basic VARMA models with autoregressive order of 4 and moving average order of 4 , based on the findings from individual patient ARIMA models of first order differenced ICP, MAP and $\mathrm{PbtO}_{2}$ data, for each patient, confirming that such VARMA orders would encompass the variation seen in optimal ARIMA structure for ICP, MAP and $\mathrm{PbtO}_{2}$ across the population. The coefficients derived from these VARMA models were then employed to derive impulse response function (IRF) plots between: ICP and MAP, $\mathrm{PbtO}_{2}$ and MAP, and $\mathrm{PbtO}_{2}$ and ICP. The IRF plots provide a descriptive graphical representation of the impact of one physiologic parameter on another, by using the generated VARIMA model and modelling a one standard deviation orthogonal impulse of one variable on the other, and vice versa. The plots depict how much from baseline the standard error of one variable fluctuates in response to the orthogonal impulse of the other variable, and how many lags in time it takes to recover back to baseline. Similarly, tri-variate VARIMA models were created to evaluate the concurrent relationship between ICP, MAP and $\mathrm{PbtO}_{2}$, with IRF plots generated for each patient.

Finally, the influence of slow-waves of ICP, MAP and $\mathrm{PbtO}_{2}$ on one another over time were assessed using Granger causality testing of stationary first order differenced data $[29,30,34]$. This was tested in every patient. For Granger causality, both $F$-test statistic value and $p$-values were recorded, with alpha set at 0.05 . We did not correct for multiple comparisons.

\section{Results}

\subsection{Patient characteristics}

A total of 47 patients were included in this study with highfrequency ICP, MAP and $\mathrm{PbtO}_{2}$ physiology. The median age was 45 years (IQR 31 to 62 years), admission total GCS was 6 (IQR 3 to 10), and the admission GCS motor score was 3 (IQR 1 to 5). The median length of overall physiologic recording was $136.1 \mathrm{~h}$ (IQR 88.3 to $174.5 \mathrm{~h}$ ). For those with recorded data, seven patients had bilaterally unreactive pupils, three unilateral unreactive pupil and 32 had normal pupils. Eight patients suffered pre-hospital hypoxic episodes, while 4 suffering a hypotensive episode. The median admission Marshall CT grade was 3 (IQR 2 to 6), while the median admission Rotterdam CT score was 3 (IQR 3 to 5). Finally, 35 patients had traumatic subarachnoid hemorrhage on admission CT, while 15 had an epidural hematoma present.

\subsection{ARIMA structure of ICP, MAP and $\mathrm{PbtO}_{2}$}

Appendix A of the Supplementary Materials provides the ARIMA models tables for each patient, for ICP, MAP and $\mathrm{PbtO}_{2}$. The ARIMA structure was assessed on first order differenced data, from the first 5 days of physiologic recording. Each patient displayed varying optimal ARIMA orders for ICP, MAP and $\mathrm{PbtO}_{2}$ in keeping with individual patient heterogeneity in physiologic behavior. However, the trend seen across all 47 patients was that ICP and MAP slow-wave appeared to have similar optimal ARIMA modal structure for a given patient, while $\mathrm{PbtO}_{2}$ displayed a different optimal model structure with much higher autoregressive and moving average orders. This suggests that the time-series 
behavior of $\mathrm{PbtO}_{2}$ slow-waves is much different than that of ICP and MAP.

\subsection{ICP, MAP and $\mathrm{PbtO}_{2}$ slow-wave time series analysis}

To facilitate exploration of the relationship between slowwave fluctuations in ICP, MAP and $\mathrm{PbtO}_{2}$, we assessed Granger causality between the slow-waves each physiological parameter, employed VARIMA modelling of the bivariate relationships, and then the tri-variate relationship.

\subsection{Granger causality testing of slow-waves}

To assess is there was a causal direction in the relationship between slow-waves of ICP and MAP, $\mathrm{PbtO}_{2}$ and MAP, and ICP and $\mathrm{PbtO}_{2}$, Granger causality testing was performed on the slow-wave physiologic data. In general, for the majority of patients testing slow-waves of ICP and MAP, MAP displayed a causal relation on ICP, as demonstrated by the larger F-statistic value favoring the MAP on ICP directionality. Similarly, for the majority of patients, MAP displayed a causal directional relationship on $\mathrm{PbtO}_{2}$ slow waves. Finally, for the majority of patients, ICP displayed causal impact on $\mathrm{PbtO}_{2}$ slow-waves. Of note, for most patients, the magnitude of the $F$-statistic values was much higher for MAP on ICP versus $\mathrm{MAP}$ on $\mathrm{PbtO}_{2}$ causal relationship. Appendix B provides the Granger causality testing for each patient, with the $F$-statistic and $p$-value reported.

\subsection{ICP versus MAP, $\mathrm{PbtO}_{2}$ versus MAP, and $\mathrm{PbtO}_{2}$ versus ICP VARIMA models}

VARIMA models with autoregressive orders of 4 , integrative/differencing order of 1 , and moving average order of 4 , were generated for each patient, for: ICP and MAP, $\mathrm{PbtO}_{2}$ and MAP, and $\mathrm{PbtO}_{2}$ and ICP. With these models, IRF plots were generated for each patient, for each physiologic relationship. Figure 1 displays a patient example of the IRF plots for: ICP and MAP, $\mathrm{PbtO}_{2}$ and MAP, and $\mathrm{PbtO}_{2}$ and ICP. The IRF plots allow for visual assessment of the relationship between two physiologic variables, evaluating the impact of one standard deviation impulse of one physiologic variable on the other, and vice versa. For all patients, the IRF plots for MAP and ICP demonstrated a definite response of ICP to MAP. However, most patients demonstrated an attenuated response of $\mathrm{PbtO}_{2}$ to MAP or ICP impulses on IRF plots. Particularly the response of $\mathrm{PbtO}_{2}$ to MAP, where there is minimal positive change in the IRF plot, and perhaps a very extended and slow return of $\mathrm{PbtO}_{2}$ to baseline. This suggests that the $\mathrm{PbtO}_{2}$ response to MAP or ICP slow-wave fluctuations is limited, with the $\mathrm{PbtO}_{2}$ response to MAP occurring well past $5 \mathrm{~min}$ in duration based off of the IRF plots.

\subsection{ICP, MAP and $\mathrm{PbtO}_{2}$ VARIMA models}

Running tri-variate VARIMA models using slow-waves of ICP, MAP and $\mathrm{PbtO}_{2}$, we generated IRF plots for each patient. Figure 2 provides a patient example of an IRF plot. For the majority of patients evaluated, these tri-variate models and IRF plots confirmed the relationships seen in the bivariate VARIMA modelling. MAP demonstrated a definite response on ICP. However, ICP and MAP failed to elicit any significant fluctuations in $\mathrm{PbtO}_{2}$, again suggesting that $\mathrm{PbtO}_{2}$ fluctuations are not occurring or responding to slow-wave perturbations in MAP or ICP, commonly associated with cerebral autoregulation. Furthermore, the slight positive response of $\mathrm{PbtO}_{2}$ to MAP seen on IRF, extends far beyond the 5-min lag mark, reinforcing that any slight response that is seen in $\mathrm{PbtO}_{2}$ to MAP may be well outside of the response seen with cerebral autoregulation.

\section{Discussion}

Using the multi-center prospectively collected CENTERTBI HR ICU cohort, we have been able to investigate the time-series relationships between slow-waves of ICP, MAP and $\mathrm{PbtO}_{2}$. Overall, we have shown that $\mathrm{PbtO}_{2}$ does not appear to respond as ICP and MAP do to slow-wave fluctuations. This raises the question of the utility of $\mathrm{PbtO}_{2}$ in the derivation of cerebrovascular reactivity indices. Some important features regarding the relationship between slowwaves of these physiologic parameters deserve highlighting.

First, upon evaluating the optimal ARIMA time-series structure of ICP, MAP and $\mathrm{PbtO}_{2}$ slow-waves, it was clear from all patients, that the behavior of $\mathrm{PbtO}_{2}$ is much different than that of ICP and MAP. Though these results are preliminary, they suggest that $\mathrm{PbtO}_{2}$ does not appear to behave as ICP and MAP do to slow-wave perturbations, on a time scale associated with cerebrovascular reactivity/cerebral autoregulation. This is important, as it raises the question of whether $\mathrm{PbtO}_{2}$ signals should be used in the derivation of cerebrovascular reactivity indices such as ORx. This is probably related to a slow response time of Licox electrode readings- this way $\mathrm{B}$ waves of oxygenation are eliminated. In contrast, they can be seen in NIRS-derived brain mixed blood oxygenation [35].

Second, Granger causality analysis confirmed the strong directional relationship of MAP on ICP, as seen in previous work on the topic [29, 30, 34]. A similar directional relationship was seen on Granger testing for MAP on $\mathrm{PbtO}_{2}$, though the magnitude of the $F$-statistic was much smaller than that see for the MAP on ICP relationship. This implies that though MAP does appear to influence $\mathrm{PbtO}_{2}$ during periods of targeted MAP treatment, as seen in clinical intervention studies [2, 3], as the MAP on ICP relationship 
appears to be stronger. This is important, as it implies the MAP/ICP derived indices may provide more reliable information regarding the cerebrovascular vasogenic response, versus $\mathrm{MAP} / \mathrm{PbtO}_{2}$. This of course carries implications for the calculation of $\mathrm{PbtO}_{2}$ derived indices, like ORx. These results corroborate the previous findings in the literature that have failed to document strong associations between ORx and ICP-derived cerebrovascular reactivity indices $[11,12]$. It must be acknowledge, however, that in the setting of active and well controlled MAP/CPP in TBI care, the variations in MAP may not be sufficient enough to see a reliable and direct influence on $\mathrm{PbtO}_{2}$. Other small cohort studies have documented the impact of CPP (and thus MAP) changes on recorded $\mathrm{PbtO}_{2}$ [36]. If much larger fluctuations in MAP were seen, perhaps there would have been a more robust impact on the $\mathrm{PbtO}_{2}$ values seen in our cohort.

Third, applying bi-variate and tri-variate VARIMA models, we were able to derive IRF plots to aid in the evaluation of the response of ICP and $\mathrm{PbtO}_{2}$ to changes in MAP. As highlighted in Figs. 1 and 2, the population displayed a characteristic response of ICP slow-waves to one standard deviation impulse in MAP slow-waves, as seen in our previous work on ICP and MAP time-series [29, 30]. However, the response of $\mathrm{PbtO}_{2}$ slow-waves to both MAP and ICP slow-wave impulses, was virtually non-existent. This lack of response in $\mathrm{PbtO}_{2}$ slow-waves to impulses in ICP and MAP slow-waves, further raises the question of the utility of $\mathrm{PbtO}_{2}$ in the derivation of cerebrovascular reactivity indices.

In general, based on this preliminary exploratory analysis, there should be caution when using $\mathrm{PbtO}_{2}$ in the derivation of cerebrovascular reactivity indices. From previous literature it is becoming clearer that ORx displays little-tono association with other multi-modal monitoring derived cerebrovascular reactivity indices $[11,12]$. This includes ICP and NIRS based metrics which have some experimental literature to support their ability to measure aspects of the Lassen curve [14-17]. This current work corroborates those findings, using time-series analysis of slow-waves in ICP, MAP and $\mathrm{PbtO}_{2}$. These findings should not be of great surprise, given that $\mathrm{PbtO}_{2}$ is a measure of extracellular oxygen diffusion [5, 18], and may not respond in the same frequency range compared to ICP and MAP.

However, it must be acknowledged, that these findings in no way detract from the literature supporting the association between $\mathrm{PbtO}_{2}$ and $\mathrm{ORx}$, with long-term outcomes in TBI $[2,3,7,8]$. $\mathrm{PbtO}_{2}$ derived indices, however taking into account much slower fluctuations in raw physiology and waves than those raw signals utilized for PRx calculation, may still provide important prognostic information in TBI, and thus should not be discounted. We merely suggest that using them for the interpretation of cerebrovascular reactivity, or derivation of individualized CPP targets, should be done so with caution at this time. The results from the phase
II randomized control trial comparing therapy directed at $\mathrm{PbtO}_{2}$ and ICP, versus ICP alone, confirm the role of $\mathrm{PbtO}_{2}$ in TBI care [2]. These results have subsequently sparked the ongoing phase III study, the Brain Oxygen Optimization in Traumatic Brain Injury III (BOOST-3) trial, and have received support from the recent international Seattle consensus meeting regarding TBI care guidelines [37, 38].

\subsection{Limitations}

Despite the important preliminary findings in this study, there exist some limitations which deserve highlighting. First, this is an entirely exploratory analysis into the statistical time-series properties of ICP, MAP and $\mathrm{PbtO}_{2}$ slowwaves in a small cohort of 47 patients. Thus, the results from this analysis should remain exploratory in nature, until further validation occurs. With that said, the findings here are supported from previous analysis of ORx and its association with other cerebrovascular reactivity indices in TBI [11, 12].

Second, this cohort is one that underwent active treatment for ICP and CPP during the course of their ICU care. Thus, any administered therapies could have potentially impacted the relationships between the recorded signals and their slow-waves. Furthermore, we did not have high temporal sampling of $\mathrm{PaO}_{2}$ in this cohort. Thus, the relationship between arterial oxygen content and $\mathrm{PbtO}_{2}$ could not be commented on in this study. It is well known that $\mathrm{PbtO}_{2}$ levels are influenced by a variety of factors involved in oxygen uptake, transport and end-organ delivery, from the alveolar-capillary interface in the lungs, all the way to the blood-brain-barrier. Thus, anything which interferes with oxygen delivery to the alveolar-capillary interface, diffusion in the lungs, hemoglobin concentration/binding, delivery to the blood-brain-barrier (ie. cardiac output/cerebral blood flow), diffusion into the cerebral extracellular space, and endorgan use within the respiratory chain of oxidative metabolism, may have a direct impact on the recorded $\mathrm{PbtO}_{2}$ levels. In particular, the relationship between $\mathrm{PaO}_{2}$ and $\mathrm{PbtO}_{2}$ is well documented, were $\mathrm{PaO}_{2}$ levels below $\sim 100 \mathrm{mmHg}$ have been shown to directly impact recorded $\mathrm{PbtO}_{2}$ [36]. This and other systemic aspects which impact $\mathrm{PbtO}_{2}$ were not accounted for within this small exploratory study. However, again, despite this, the findings of our work are corroborated by previous literature on the topic.

Finally, we used 10-s resolution data for ICP, MAP and $\mathrm{PbtO}_{2}$. As mentioned, $\mathrm{PbtO}_{2}$ is a measure of extracellular oxygen diffusion, and subject to much slower frequency of physiologic response times compared to raw ICP and MAP. As such, some studies in TBI have used long windows of data in the derivation of ORx [20]. We did not evaluate the impact of window length in this study, as such large data windows evaluate ultra-low frequency below the optimal frequency ranges associated with cerebrovascular reactivity $[39,40]$. At 

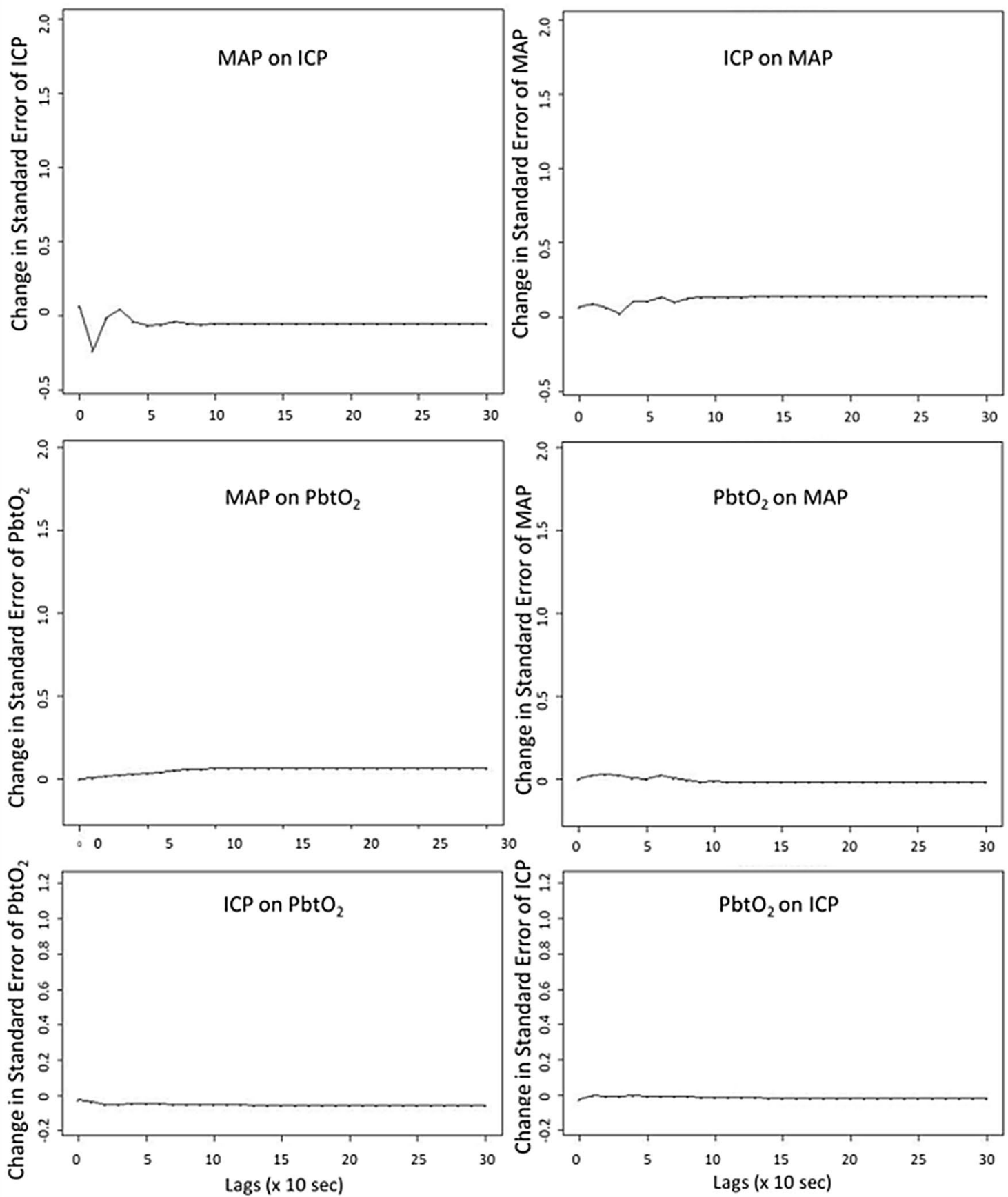

Fig. 1 VARIMA IRF Plots for ICP/MAP, $\mathrm{PbtO}_{2} / \mathrm{MAP}$, and $\mathrm{PbtO}_{2}$ / ICP-Patient Example. ICP intracranial pressure, IRF impulse response function, lags refers to number of time points where 1 lag is $10-\mathrm{s}, \mathrm{MAP}$ mean arterial pressure, $\mathrm{PbtO}_{2}$ brain tissue oxygen, $V A R$ $I M A$ vector autoregressive integrative moving average. All VARIMA models were constructed using an autoregressive order of 4 , integra-

this point in time, it is uncertain if such metrics derived from long window lengths, or lower temporal resolution data, carry any information regarding cerebrovascular reactivity [29]. Consequently, we focused on 10-s-by-10-s slow-wave data. tive order of 1 , and moving average order of 4 . The IRF plots display the response of one physiologic variable to one standard deviation impulse of the other. Of note, there is minimal response of $\mathrm{PbtO}_{2}$ to an impulse in MAP or ICP, with a quite extended duration low level elevation in $\mathrm{PbtO}_{2}$ to MAP impulse that fails to return to baseline even after 5 min worth of lags

As the goal of this study was to report the statistical relationship over time of raw recorded cerebral physiology, we did not focus on the derivation of cerebrovascular reactivity indices, and comparing between different groups based on the values of these indices. The cohort too small for 

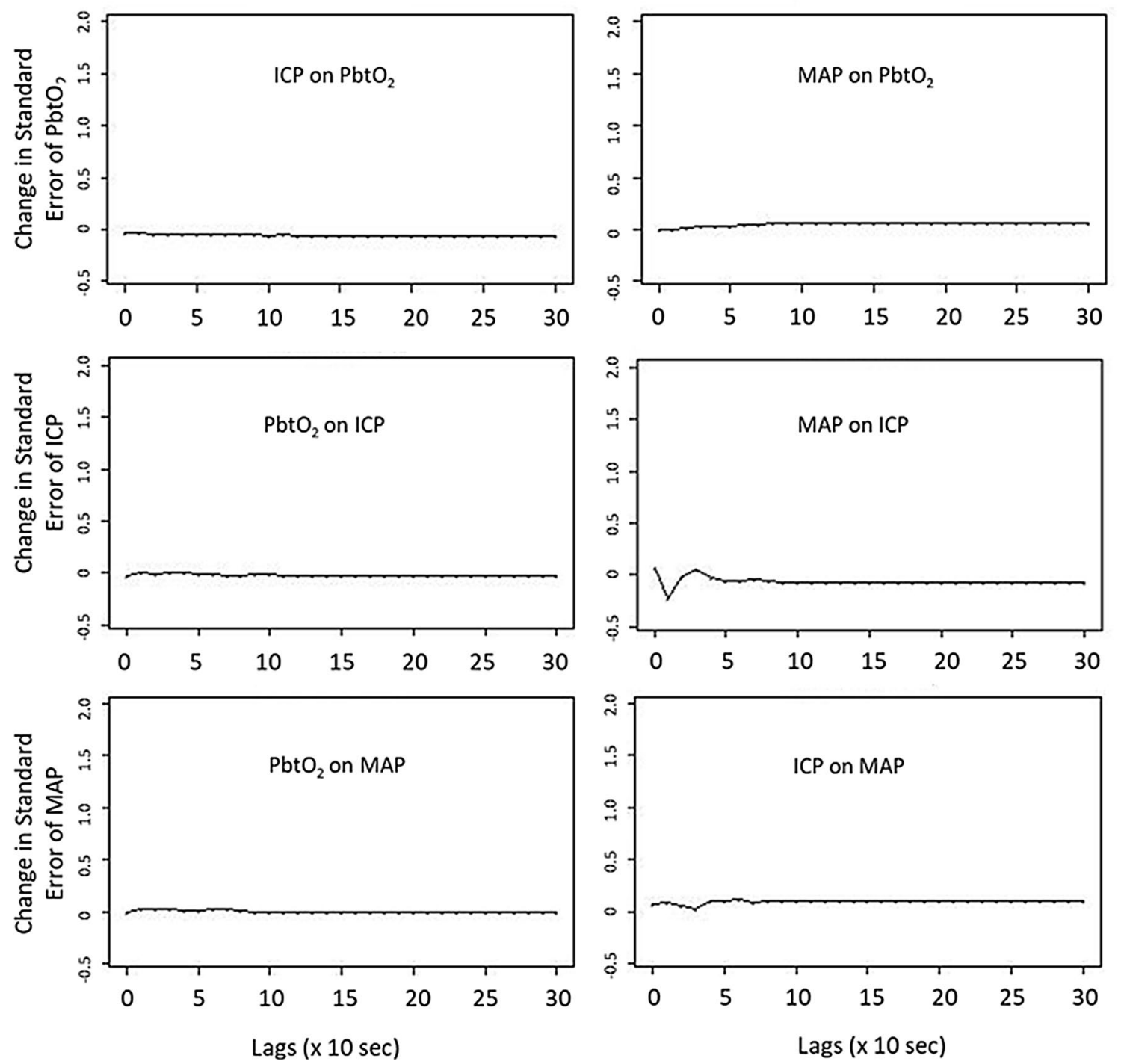

Fig. 2 Tri-Variate VARIMA Model IRF Plot-ICP, MAP and $\mathrm{PbtO}_{2}$-Patient Example. ICP intracranial pressure, IRF impulse response function, lags refers to number of time points where 1 lag is 10 -s, MAP mean arterial pressure, $\mathrm{PbtO}_{2}$ brain tissue oxygen, $V A R$ $I M A$ vector autoregressive integrative moving average. The tri-vari-

subgroup analysis of this nature. In addition, the definition of who has "impaired" versus "intact" cerebrovascular reactivity in TBI is still unclear at this time. There are literature defined thresholds for certain indices (like PRx), but these are based on association with 6-month dichotomized clinical outcomes [41, 42]. These thresholds do not necessarily indicate who does or does not have impaired cerebrovascular reactivity. With that said, we have recently evaluated the insult burden of ICP, $\mathrm{PRx}$ and $\mathrm{PbtO} 2$ in our resent publication [43]. Again, here we don't have a good definition of what defines impaired reactivity, and only are able to provide $\%$ time and dose above/below thresholds associated with global patient outcome. As such, the results of this current

ate VARIMA model was constructed using an autoregressive order of 4 , integrative order of 1 , and moving average order of 4 . The IRF plots display the response of one physiologic variable to one standard deviation impulse of the other. Of note, there is minimal response of $\mathrm{PbtO}_{2}$ to an impulse in MAP or ICP

study are to be validated in a much larger cohort of TBI patients with $\mathrm{PbtO}_{2}$ monitoring, from the emerging CAHRTBI dataset in Canada [44], with a plan to evaluate various subgroups.

The results within this study are experimental/exploratory in nature. There is a need for future validation of these results in larger cohorts with $\mathrm{PbtO}_{2}$ data. Similarly, if one wanted to focus entirely on the vasogenic frequency range associated with cerebrovascular reactivity, application of various band pass filtering techniques prior to signal decimation would allow for this. Further, altering the window length of physiology evaluation also needs to occur, as these relationships may be different depending on the time or day 
post-injury. This work is part of the planned analysis for our group and other collaborative efforts in the area of high frequency physiologic signal analysis in TBI patients. The results here provide a platform to move forward with this type of complex time-series analysis.

\section{Conclusions}

There is a reproducible relationship between slow-wave fluctuations of ICP and MAP, as demonstrated across various time-series analytic techniques. $\mathrm{PbtO}_{2}$ does not appear to reliably respond in time to slow-wave fluctuations in MAP, as demonstrated on various VARIMA models across all patients. These findings suggest that $\mathrm{PbtO}_{2}$ should not be utilized in the derivation of cerebrovascular reactivity metrics in TBI, as it does not appear to be responsive to changes in MAP slow-waves, over a time scale commonly associated with cerebrovascular reactivity.

Acknowledgements Data used in preparation of this manuscript were obtained in the context of CENTER-TBI, a large collaborative project with the support of the European Union 7th Framework program (EC Grant 602150). Additional funding was obtained from the Hannelore Kohl Stiftung (Germany), from OneMind (USA) and from Integra LifeSciences Corporation (USA).

CENTER-TBI High Resolution Sub-Study Participants and Investigators Audny Anke, Department of Physical Medicine and Rehabilitation, University hospital Northern Norway; Ronny Beer, Department of Neurology, Neurological Intensive Care Unit, Medical University of Innsbruck, Innsbruck, Austria; Bo Michael Bellander, Department of Neurosurgery \& Anesthesia \& intensive care medicine, Karolinska University Hospital, Stockholm, Sweden; Erta Beqiri, NeuroIntensive Care, Niguarda Hospital, Milan, Italy; Andras Buki, Department of Neurosurgery, Medical School, University of Pécs, Hungary and Neurotrauma Research Group, János Szentágothai Research Centre, University of Pécs, Hungary; Manuel Cabeleira, Brain Physics Lab, Division of Neurosurgery, Dept of Clinical Neurosciences, University of Cambridge, Addenbrooke's Hospital, Cambridge, UK; Marco Carbonara, Neuro ICU, Fondazione IRCCS Cà Granda Ospedale Maggiore Policlinico, Milan, Italy; Arturo Chieregato, NeuroIntensive Care, Niguarda Hospital, Milan, Italy; Giuseppe Citerio, NeuroIntensive Care Unit, Department of Anesthesia \& Intensive Care, ASST di Monza, Monza, Italy, School of Medicine and Surgery, Università Milano Bicocca, Milano, Italy; Hans Clusmann, Department of Neurosurgery, Medical Faculty RWTH Aachen University, Aachen, Germany; Endre Czeiter, Department of Neurosurgery, University of Pecs and MTA-PTE Clinical Neuroscience MR Research Group and Janos Szentagothai Research Centre, University of Pecs, Hungarian Brain Research Program (Grant No. KTIA 13 NAP-A-II/8), Pecs, Hungary; Marek Czosnyka, Brain Physics Lab, Division of Neurosurgery, Dept of Clinical Neurosciences, University of Cambridge, Addenbrooke's Hospital, Cambridge, UK; Bart Depreitere, Department of Neurosurgery, University Hospitals Leuven, Leuven, Belgium; Ari Ercole, Division of Anaesthesia, University of Cambridge, Addenbrooke's Hospital, Cambridge, UK; Shirin Frisvold, Department of Anesthesiology and Intensive care, University Hospital Northern Norway, Tromso, Norway; Raimund Helbok, Department of Neurology, Neurological Intensive Care Unit, Medical University of Innsbruck, Innsbruck, Austria; Stefan Jankowski, Neurointensive Care, Sheffield Teaching Hospitals NHS Foundation Trust,
Sheffield, UK; Danile Kondziella, Departments of Neurology, Clinical Neurophysiology and Neuroanesthesiology, Region Hovedstaden Rigshospitalet, Copenhagen, Denmark; Lars-Owe Koskinen, Department of Clinical Neuroscience, Neurosurgery, Umeå University, Umeå, Sweden; Ana Kowark, Department of Anaesthesiology, University Hospital of Aachen, Aachen, Germany; David K. Menon, Division of Anaesthesia, University of Cambridge, Addenbrooke's Hospital, Cambridge, UK; Geert Meyfroidt, Intensive Care Medicine, University Hospitals Leuven, Leuven, Belgium; Kirsten Moeller, Department Neuroanesthesiology, Region Hovedstaden Rigshospitalet, Copenhagen, Denmark; David Nelson, Department of Neurosurgery \& Anesthesia \& intensive care medicine, Karolinska University Hospital, Stockholm, Sweden; Anna Piippo-Karjalainen, Helsinki University Central Hospital, Helsinki, Finland; Andreea Radoi, Department of Neurosurgery, Vall d'Hebron University Hospital, Barcelona, Spain; Arminas Ragauskas, Department of Neurosurgery, Kaunas University of technology and Vilnius University, Vilnius, Lithuania; Rahul Raj, Helsinki University Central Hospital, Helsinki, Finland; Jonathan Rhodes, Department of Anaesthesia, Critical Care \& Pain Medicine NHS Lothian \& University of Edinburg, Edinburgh, UK; Saulius Rocka, Department of Neurosurgery, Kaunas University of technology and Vilnius University, Vilnius, Lithuania; Rolf Rossaint, Department of Anaesthesiology, University Hospital of Aachen, Aachen, Germany; Juan Sahuquillo, Department of Neurosurgery, Vall d'Hebron University Hospital, Barcelona, Spain; Oliver Sakowitz, Klinik für Neurochirurgie, Klinikum Ludwigsburg, Ludwigsburg, Germany, Department of Neurosurgery, University Hospital Heidelberg, Heidelberg, Germany; Peter Smielewski, Brain Physics Lab, Division of Neurosurgery, Dept of Clinical Neurosciences, University of Cambridge, Addenbrooke's Hospital, Cambridge, UK; Nino Stocchetti, Department of Pathophysiology and Transplantation, Milan University, and Neuroscience ICU, Fondazione IRCCS Cà Granda Ospedale Maggiore Policlinico, Milano, Italy; Nina Sundström, Department of Radiation Sciences, Biomedical Engineering, Umea University, Umea, Sweden; Riikka Takala, Perioperative Services, Intensive Care Medicine, and Pain Management, Turku University Central Hospital and University of Turku, Turku, Finland; Tomas Tamosuitis, Neuro-intensive Care Unit, Kaunas University of Health Sciences, Kaunas, Lithuania; Olli Tenovuo, Rehabilitation and Brain Trauma, Turku University Central Hospital and University of Turku, Turku, Finland; Peter Vajkoczy, Neurologie, Neurochirurgie und Psychiatrie, Charité - Universitätsmedizin Berlin, Berlin, Germany; Alessia Vargiolu, NeuroIntensive Care Unit, Department of Anesthesia \& Intensive Care, ASST di Monza, Monza, Italy; Rimantas Vilcinis, Department of Neurosurgery, Kaunas University of Health Sciences, Kaunas, Lithuania; Stefan Wolf, Department of Neurosurgery, Charité - Universitätsmedizin Berlin, corporate member of Freie Universität Berlin, Humboldt-Universität zu Berlin, and Berlin Institute of Health, Berlin, Germany; Alexander Younsi, Department of Neurosurgery, University Hospital Heidelberg, Heidelberg, Germany; Frederick A. Zeiler, Division of Anaesthesia, University of Cambridge, Addenbrooke's Hospital, Cambridge, UK, Section of Neurosurgery, Department of Surgery, Rady Faculty of Health Sciences, University of Manitoba, Winnipeg, MB, Canada.

Author contributions PS and AE were responsible for concept, design, data interpretation and manuscript preparation. MC, MC, NS, and $\mathrm{PJH}$ were responsible for data interpretation and manuscript composition. FAZ was responsible for concept, design, analysis, interpretation and manuscript composition.

Funding FAZ receives research support from the Manitoba Public Insurance (MPI) Neuroscience/TBI Research Endowment, the United States National Institutes of Health (NIH) through the National Institute of Neurological Disorders and Stroke (NINDS), the Canadian Institutes for Health Research (CIHR), the Canadian Foundation for Innovation (CFI), the University of Manitoba Centre on Aging, the 
University of Manitoba VPRI Research Investment Fund (RIF), the University of Manitoba Rudy Falk Clinician-Scientist Professorship, and the Health Sciences Centre Foundation Winnipeg. MC is supported by NIHR Cambridge BRC. PH is supported by the NIHR (Research Professorship, Cambridge BRC, Global Health Research Group on Neurotrauma) and the Royal College of Surgeons of England.

\section{Compliance with ethical standards}

Conflict of interest PS and MC receive part of licensing fees for the software ICM + (Cambridge Enterprise Ltd, UK) used for data collection and analysis in this study. MC has consultancy agreement with Integra, PS has consultancy agreements with Integra Life Sciences and Pressura Neuro Ltd.

Open Access This article is licensed under a Creative Commons Attribution 4.0 International License, which permits use, sharing, adaptation, distribution and reproduction in any medium or format, as long as you give appropriate credit to the original author(s) and the source, provide a link to the Creative Commons licence, and indicate if changes were made. The images or other third party material in this article are included in the article's Creative Commons licence, unless indicated otherwise in a credit line to the material. If material is not included in the article's Creative Commons licence and your intended use is not permitted by statutory regulation or exceeds the permitted use, you will need to obtain permission directly from the copyright holder. To view a copy of this licence, visit http://creativecommons.org/licenses/by/4.0/.

\section{References}

1. Le Roux P, Menon DK, Citerio G, Vespa P, Bader MK, Brophy G, et al. The International Multidisciplinary Consensus Conference on Multimodality Monitoring in Neurocritical Care: evidentiary tables: a statement for healthcare professionals from the Neurocritical Care Society and the European Society of Intensive Care Medicine. Neurocrit Care. 2014;21(Suppl 2):S297-361.

2. Okonkwo DO, Shutter LA, Moore C, Temkin NR, Puccio AM, Madden CJ, et al. Brain oxygen optimization in severe traumatic brain injury phase-II: a phase II randomized trial. Crit Care Med. 2017;45:1907-14.

3. Hawryluk GWJ, Phan N, Ferguson AR, Morabito D, Derugin N, Stewart CL, et al. Brain tissue oxygen tension and its response to physiological manipulations: influence of distance from injury site in a swine model of traumatic brain injury. J Neurosurg. 2016;125:1217-28.

4. Soehle M, Jaeger M, Meixensberger J. Online assessment of brain tissue oxygen autoregulation in traumatic brain injury and subarachnoid hemorrhage. Neurol Res. 2003;25:411-7.

5. Rosenthal G, Hemphill JC, Manley G. Brain tissue oxygen tension is more indicative of oxygen diffusion than oxygen delivery and metabolism in patients with traumatic brain injury. Crit Care Med. 2009;37:379-80.

6. Carney N, Totten AM, O'Reilly C, Ullman JS, Hawryluk GWJ, Bell MJ, et al. Guidelines for the management of severe traumatic brain injury, Fourth Edition. Neurosurgery. 2017;80:6-15.

7. Jaeger M, Schuhmann MU, Soehle M, Meixensberger J. Continuous assessment of cerebrovascular autoregulation after traumatic brain injury using brain tissue oxygen pressure reactivity. Crit Care Med. 2006;34:1783-8.

8. Jaeger M, Lang EW. Cerebrovascular pressure reactivity and cerebral oxygen regulation after severe head injury. Neurocrit Care. 2013;19:69-73.
9. Lang EW, Czosnyka M, Mehdorn HM. Tissue oxygen reactivity and cerebral autoregulation after severe traumatic brain injury. Crit Care Med. 2003;31:267-71.

10. Jaeger M, Dengl M, Meixensberger J, Schuhmann MU. Effects of cerebrovascular pressure reactivity-guided optimization of cerebral perfusion pressure on brain tissue oxygenation after traumatic brain injury. Crit Care Med. 2010;38:1343-7.

11. Zeiler FA, Donnelly J, Menon DK, Smielewski P, Zweifel C, Brady K, et al. Continuous autoregulatory indices derived from multi-modal monitoring: each one is not like the other. J Neurotrauma. 2017;34:3070-80.

12. Andresen M, Donnelly J, Aries M, Juhler M, Menon D, Hutchinson $\mathrm{P}$, et al. Further controversies about brain tissue oxygenation pressure-reactivity after traumatic brain injury. Neurocrit Care. 2018;28:162-8.

13. Radolovich DK, Aries MJH, Castellani G, Corona A, Lavinio A, Smielewski P, et al. Pulsatile intracranial pressure and cerebral autoregulation after traumatic brain injury. Neurocrit Care. 2011;15:379-86.

14. Zeiler FA, Donnelly J, Calviello L, Lee JK, Smielewski P, Brady $\mathrm{K}$, et al. Validation of pressure reactivity and pulse amplitude indices against the lower limit of autoregulation, part I: experimental intracranial hypertension. J Neurotrauma. 2018;35:2803-11.

15. Zeiler FA, Lee JK, Smielewski P, Czosnyka M, Brady K. Validation of intracranial pressure-derived cerebrovascular reactivity indices against the lower limit of autoregulation, part II: experimental model of arterial hypotension. J Neurotrauma. 2018;35:2812-9.

16. Brady KM, Lee JK, Kibler KK, Easley RB, Koehler RC, Shaffner $\mathrm{DH}$. Continuous measurement of autoregulation by spontaneous fluctuations in cerebral perfusion pressure: comparison of 3 methods. Stroke. 2008;39:2531-7.

17. Lee JK, Kibler KK, Benni PB, Easley RB, Czosnyka M, Smielewski $\mathrm{P}$, et al. Cerebrovascular reactivity measured by nearinfrared spectroscopy. Stroke. 2009;40:1820-6.

18. Kohler K, Nallapareddy S, Ercole A. In silico model of critical cerebral oxygenation after traumatic brain injury: implications for rescuing hypoxic tissue. J Neurotrauma. 2019;36:2109.

19. Czosnyka M, Smielewski P, Kirkpatrick P, Laing RJ, Menon D, Pickard JD. Continuous assessment of the cerebral vasomotor reactivity in head injury. Neurosurgery. 1997;41:11-7 discussion 17-19.

20. Zeiler FA, Donnelly J, Calviello L, Smielewski P, Menon DK, Czosnyka M. Pressure autoregulation measurement techniques in adult traumatic brain injury, part II: a scoping review of continuous methods. J Neurotrauma. 2017;34:3224-377.

21. Budohoski KP, Zweifel C, Kasprowicz M, Sorrentino E, Diedler J, Brady KM, et al. What comes first? The dynamics of cerebral oxygenation and blood flow in response to changes in arterial pressure and intracranial pressure after head injury. Br J Anaesth. 2012;108:89-99.

22. Maas AIR, Menon DK, Steyerberg EW, Citerio G, Lecky F, Manley GT, et al. Collaborative European NeuroTrauma Effectiveness Research in Traumatic Brain Injury (CENTER-TBI): a prospective longitudinal observational study. Neurosurgery. 2015;76:67-80.

23. Vande Vyvere T, Wilms G, Claes L, Martin Leon F, Nieboer D, Verheyden J, et al. Central versus local radiological reading of acute computed tomography characteristics in multi-center traumatic brain injury research. J Neurotrauma. 2019;36:1080-92.

24. Marshall LF, Marshall SB, Klauber MR, Van Berkum CM, Eisenberg H, Jane JA, et al. The diagnosis of head injury requires a classification based on computed axial tomography. J Neurotrauma. 1992;9(Suppl 1):S287-292.

25. Maas AIR, Hukkelhoven CWPM, Marshall LF, Steyerberg EW. Prediction of outcome in traumatic brain injury with computed tomographic characteristics: a comparison between the computed 
tomographic classification and combinations of computed tomographic predictors. Neurosurgery. 2005;57:1173-82 discussion 1173-1182.

26. Doiron D, Marcon Y, Fortier I, Burton P, Ferretti V. Software Application Profile: opal and Mica: open-source software solutions for epidemiological data management, harmonization and dissemination. Int J Epidemiol. 2017;46:1372-8.

27. Zeiler FA, Smielewski P, Donnelly J, Czosnyka M, Menon DK, Ercole A. Estimating pressure reactivity using noninvasive Doppler-based systolic flow index. J Neurotrauma. 2018;35:1559-688.

28. Zeiler FA, Smielewski P, Stevens A, Czosnyka M, Menon DK, Ercole A. Non-invasive pressure reactivity index using Doppler systolic flow parameters: a pilot analysis. J Neurotrauma. 2019;36:713-20.

29. Thelin EP, Raj R, Bellander B-M, Nelson D, Piippo-Karjalainen A, Siironen J, et al. Comparison of high versus low frequency cerebral physiology for cerebrovascular reactivity assessment in traumatic brain injury: a multi-center pilot study. J Clin Monit Comput. 2019. https://doi.org/10.1007/s10877-019-00392-y.

30. Zeiler FA, Aries M, Cabeleira M, van Essen T, Stocchetti N, Menon D, et al. Statistical cerebrovascular reactivity signal properties after secondary decompressive craniectomy in traumatic brain injury: a CENTER-TBI pilot analysis. J Neurotrauma. 2020. https://doi.org/10.1089/neu.2019.6726.

31. Chatfield C. The analysis of time series: an introduction. 6th ed. Boca Raton: Chapman and Hall/CRC; 2016.

32. Lutkepohl $\mathrm{H}$. New introduction to multiple time series analysis. 2nd ed. Berlin: Springer; 2010.

33. Douc R, Moulines E, Stoffer D. Nonlinear time series: theory, methods and applications with R examples. 1st ed. Boca Raton: Chapman and Hall/CRC; 2014.

34. Gao L, Smielewski P, Czosnyka M, Ercole A. Early asymmetric cardio-cerebral causality and outcome after severe traumatic brain injury. J Neurotrauma. 2017;34:2743-52.

35. Diedler J, Zweifel C, Budohoski KP, Kasprowicz M, Sorrentino E, Haubrich C, et al. The limitations of near-infrared spectroscopy to assess cerebrovascular reactivity: the role of slow frequency oscillations. Anesth Analg. 2011;113:849-57.

36. Dellazizzo L, Demers S-P, Charbonney E, Williams V, Serri K, Albert M, et al. Minimal PaO2 threshold after traumatic brain injury and clinical utility of a novel brain oxygenation ratio. $\mathrm{J}$ Neurosurg. 2018;131:1-9.
37. Chesnut R, Aguilera S, Buki A, Bulger E, Citerio G, Cooper DJ, et al. A management algorithm for adult patients with both brain oxygen and intracranial pressure monitoring: the Seattle International Severe Traumatic Brain Injury Consensus Conference (SIBICC). Intensive Care Med. 2020;46:919.

38. Hawryluk GWJ, Aguilera S, Buki A, Bulger E, Citerio G, Cooper DJ, et al. A management algorithm for patients with intracranial pressure monitoring: the Seattle International Severe Traumatic Brain Injury Consensus Conference (SIBICC). Intensive Care Med. 2019;45:1783-94.

39. Howells T, Johnson U, McKelvey T, Enblad P. An optimal frequency range for assessing the pressure reactivity index in patients with traumatic brain injury. J Clin Monit Comput. 2015;29:97-105.

40. Fraser CD, Brady KM, Rhee CJ, Easley RB, Kibler K, Smielewski $\mathrm{P}$, et al. The frequency response of cerebral autoregulation. J Appl Physiol Bethesda. 1985;2013(115):52-6.

41. Sorrentino E, Diedler J, Kasprowicz M, Budohoski KP, Haubrich C, Smielewski P, et al. Critical thresholds for cerebrovascular reactivity after traumatic brain injury. Neurocrit Care. 2012;16:258-66.

42. Zeiler FA, Donnelly J, Smielewski P, Menon DK, Hutchinson PJ, Czosnyka M. Critical thresholds of intracranial pressure-derived continuous cerebrovascular reactivity indices for outcome prediction in noncraniectomized patients with traumatic brain injury. $\mathrm{J}$ Neurotrauma. 2018;35:1107-15.

43. Zeiler FA, Beqiri E, Cabeleira M, Hutchinson PJ, Stocchetti N, Menon DK, et al. Brain tissue oxygen and cerebrovascular reactivity in traumatic brain injury: a CENTER-TBI exploratory analysis of insult burden. J Neurotrauma. 2020. https://doi.org/10.1089/ neu.2020.7024.

44. Bernard F, Gallagher C, Griesdale D, Kramer A, Sekhon M, Zeiler FA. The Canadian High-Resolution Traumatic Brain Injury (CAHR-TBI) Research Collaborative. Can J Neurol Sci J Can Sci Neurol. 2020;16:1-20.

Publisher's Note Springer Nature remains neutral with regard to jurisdictional claims in published maps and institutional affiliations.

\section{Affiliations}

\section{Frederick A. Zeiler ${ }^{1,2,3,4,5}$ - Manuel Cabeleira ${ }^{6} \cdot$ Peter J. Hutchinson ${ }^{7} \cdot$ Nino Stocchetti $^{8,9}$ (D) $\cdot$ Marek Czosnyka $^{6,10}$. Peter Smielewski ${ }^{6}$. Ari Ercole ${ }^{1}$ - the CENTER-TBI High-Resolution ICU (HR ICU) Sub-Study Participants and Investigators}

Manuel Cabeleira

mc916@cam.ac.uk

Peter J. Hutchinson

pjah2@cam.ac.uk

Nino Stocchetti

nino.stocchetti@policlinico.mi.it

Marek Czosnyka

mc141@medschl.cam.ac.uk

Peter Smielewski

ps10011@cam.ac.uk

Ari Ercole

ae105@cam.ac.uk
1 Division of Anaesthesia, Addenbrooke's Hospital, University of Cambridge, Cambridge, UK

2 Department of Surgery, Rady Faculty of Health Sciences, University of Manitoba, Winnipeg, MB R3A 1R9, Canada

3 Department of Human Anatomy and Cell Science, Rady Faculty of Health Sciences, University of Manitoba, Winnipeg, Canada

4 Biomedical Engineering, Faculty of Engineering, University of Manitoba, Winnipeg, Canada

5 Centre on Aging, University of Manitoba, Winnipeg, Canada 
6 Brain Physics Laboratory, Division of Neurosurgery, Dept of Clinical Neurosciences, Addenbrooke's Hospital, University of Cambridge, Cambridge CB2 0QQ, UK

7 Division of Neurosurgery, Department of Clinical Neurosciences, Addenbrooke's Hospital, University of Cambridge, Cambridge CB2 0QQ, UK

8 Neuro ICU Fondazione IRCCS Cà Granda Ospedale Maggiore Policlinico, Milan, Italy
Department of Physiopathology and Transplantation, Milan University, Milan, Italy

10 Institute of Electronic Systems, Warsaw University of Technology, Warsaw, Poland 